\title{
Non-oscillatory Finite Compact Scheme for the Equations of Ideal Magnetohydrodynamics
}

\author{
Liu Li ${ }^{\mathrm{a}}$, Shen Yiqing ${ }^{\mathrm{b}}$ \\ Key Laboratory of High Temperature Gas Dynamics, Institute of Mechanics, Chinese Academy of \\ Sciences, No.15 Beisihuanxi Road, Beijing 100190, China \\ aliuli2@imech.ac.cn, byqshen@imech.ac.cn
}

Keywords: Ideal magnetohydrodynamics, Weighted essentially non-oscillatory scheme, Finite compact scheme.

\begin{abstract}
Although the equations of ideal Magnetohydrodynamics (MHD) is a non-strictly hyperbolic system, they have a wave-like structure analogous to that of the hydrodynamics equations, various numerical schemes for hydrodynamics equations have been extended to solve the MHD equations. The finite compact (FC) scheme treats the discontinuity as the internal boundary and avoids the global dependence of the traditional compact schemes. By using a parameter-free shock detecting method, the computational domain is divided into a series of smooth regions and shock wave regions. In the shock wave regions, the shock capturing scheme is used to construct the numerical flux, and in the smooth regions the compact scheme is used, the flux of shock wave region is automatically the boundary formulation of the compact scheme. Hence, the FC scheme can resolve shock essentially non-oscillatory and achieve high order of accuracy in smooth region. This paper develops the non-oscillation finite compact scheme for the ideal MHD equations.
\end{abstract}

\section{Introduction}

The application of Magnetohydrodynamics(MHD) is received more attention recently, especially in designing thermal protection system, capturing inlet flow and improving the efficiency of combustion chambers. Since the equations of ideal Magnetohydrodynamics (MHD) have a wave-like structure analogous to that of the hydrodynamics equations, various numerical schemes for hydrodynamics equations have been extended to solve the MHD equations [1-8].

The ideal MHD equation is a non-strictly hyperbolic system. There are seven different eigenvalues of its Jacobian matrix. Hence the eigenstructure of MHD system is very complex. This results in the complexity of those numerical methods based on the eigenstructure[1-6]. The convective upwind and split pressure(CUSP) schemes, which consider the convective upwind characteristics of flow and do not require the eigen-decomposition process, have been extended to calculate the MHD equations recently[7,8].

Weighted essentially non-oscillatory(WENO) schemes have high order accuracy in smooth region and essentially no oscillation near shocks[9,10]. However, for the short waves or complex multi-scale flows, WENO schemes exhibit excessive diffusion[11,12]. In order to reduce numerical diffusion in complex flows, many hybrid schemes are proposed, such as hybrid central-WENO scheme, hybrid compact WENO scheme. Shen et al.[13-15] propose the finite compact scheme, which treats the discontinuity as the internal boundary and avoids the implicit global dependence of the traditional compact schemes. Combined with a parameter-free shock detector[15], the FC schemes show excellent advantages, i.e., the compact schemes' spectral-like resolution in smooth region and essentially non-oscillation of WENO schemes.

In this paper, combined the Energy-CUSP(E-CUSP) scheme and the finite compact reconstruction, we develop the efficient and high order accuracy low diffusion method for calculating the MHD equations. 


\section{The numerical scheme}

Governing equations. The governing equations of ideal MHD can be expressed in vector form,

$$
\frac{\partial \mathrm{U}}{\partial t}+\nabla \cdot \mathrm{F}=0
$$

where,

$$
\begin{gathered}
\mathrm{U}=\{\rho, \rho \mathrm{V}, \mathrm{B}, \rho e\}^{T}, \\
\mathrm{~F}=\left\{\rho \mathrm{V}, \rho \mathrm{VV}+p_{t} \mathrm{I}-\mathrm{BB}, \mathrm{VB}-\mathrm{BV},\left(\rho e+p_{t}\right) \mathrm{V}-\mathrm{B}(\mathrm{V} \cdot \mathrm{B})\right\}^{T} .
\end{gathered}
$$

E-CUSP schemes. In E-CUSP method[8], the $\mathrm{x}$-direction flux $\mathrm{E}$ is decomposed to convective and generalized pressure fluxes as following,

where,

$$
\mathrm{E}=\mathrm{f} u+\mathrm{P}+\varphi u
$$

$$
\begin{gathered}
\mathrm{f}=\left\{\rho, \rho u, \rho v, \rho w, \rho e, B_{x}, B_{y}, B_{z}\right\}^{T}, \varphi=\left\{0,0,0,0, p_{t}, 0,0,0\right\}^{T}, \\
\mathrm{P}=\left\{0, p_{t}-B_{x} B_{y},-B_{y} B_{x},-B_{z} B_{x},-B_{x}\left(u B_{x}+v B_{y}+w B_{z}\right),-u B_{x},-v B_{y},-w B_{z}\right\}^{T} .
\end{gathered}
$$

The numerical flux $E_{i+1 / 2}$ is constructed as,

$$
E_{1 / 2}=a_{1 / 2}\left[C^{+} f_{L}+C^{-} f_{R}\right]+\left[D_{L}^{+} P_{L}+D_{R}^{-} P_{R}\right]+\varphi_{1 / 2}
$$

where,

$$
\begin{gathered}
M_{L, R}=\frac{u_{L, R}}{a_{1 / 2}}, C^{ \pm}=\alpha_{L, R}^{ \pm}\left(1+\beta_{L, R}\right) M_{L, R} \mp \frac{1}{4} \beta_{L, R}\left(M_{L, R} \pm 1\right)^{2}, \\
\alpha_{L, R}^{ \pm}=\frac{1}{2}\left[1 \pm \operatorname{sign}\left(M_{L, R}\right)\right], \varphi_{1 / 2}=\alpha_{1 / 2}\left(C^{+}+C^{-}\right)\left(D^{+} \varphi_{L}+D^{-} \varphi_{R}\right), \\
\beta_{L, R}=-\max \left[0,1-\operatorname{int}\left(\left|M_{L, R}\right|\right)\right], D_{L, R}^{ \pm}=\alpha_{L, R}^{ \pm}\left(1+\beta_{L, R}\right)-\frac{1}{2} \beta_{L, R}\left(1 \pm M_{L, R}\right) .
\end{gathered}
$$

Non-oscillatory finite compact scheme. In paper [15], Shen and Zha proposed a lemma to detect the non-smooth stencil in computational region, i.e.,

Lemma 1. If $\tau_{5}>\min \left(I S_{0}, I S_{1}, I S_{2}\right)$, then $S^{5}=\left(x_{i-2}, x_{i-1}, \ldots, x_{i+2}\right)$ is a non-smooth stencil.

Where, $\tau_{5}=\left|I S_{0}-I S_{2}\right|$ and $I S_{0}, I S_{1}, I S_{2}$ are smoothness indicators given by Jiang and Shu[9]. There is no any artificial parameter introduced, hence this shock detecting method is robust and generally applicable. Using this lemma, the reconstruction algorithm of the finite compact is constructed as the following[15],

BEGIN

Step 0. Calculate $u_{1 / 2}$ and $u_{N+1 / 2}$ using boundary formula.

Step 1. $M=1 \quad$ ! Looking for starting and ending points for compact scheme using Lemma 1.

$$
\text { DO } i=1, N-1
$$

Calculate $I S_{0}, I S_{1}, I S_{2}$. 
END IF

$$
\begin{gathered}
\tau_{5}=\left|I S_{0}-I S_{2}\right| \\
\operatorname{IF} \tau_{5}>\min \left(I S_{0}, I S_{1}, I S_{2}\right) \text { THEN } \\
\text { end_point }(M)=i \quad \text { Compact stencil ending point. } \\
M=M+1 \\
\text { start_point }(M)=i+1 \\
\text { Calculate } u_{i+1 / 2} \text { using WENO scheme: } \\
u_{i+1 / 2}=\sum_{k=0}^{2} \omega_{k} \hat{f}^{k}\left(x_{i+1 / 2}\right)
\end{gathered}
$$

\section{ENDDO}

$$
\text { end_point }(M)=N
$$

Step 2. DO $k=1, M \quad$ ! Calculate fluxes on compact stencil (smooth region) $k$ using a compact scheme.

$$
\text { DO } i=\operatorname{start} \_ \text {point }(k) \text {, end_point }(k)-1
$$

Calculate the right hand side $d_{i+1 / 2}$ of compact scheme.

\section{ENDDO}

Solve tridiagomal compact scheme:

$$
\alpha u_{i-1 / 2}+\gamma u_{i+1 / 2}+\beta u_{i+3 / 2}=d_{i+1 / 2}
$$

Where, $i=$ start_point $(k), \ldots$, end point $(k)-1$

\section{ENDDO}

\section{END}

\section{Numerical results}

In this paper, the fifth-order WENO-Z scheme [10] and the sixth-order Pade compact scheme(parameters and formula in Eq.(5) can be found in $[15,16])$ are used in FC scheme, and the third-order TVD Rung-Kutta method[17] is used for the time marching.

Circularly polarized Alfven waves. The propagation of a monochromatic circularly polarized Alfven wave is used to test the accuracy of the FC scheme in smooth MHD flow. Computational conditions are same as in Refs.[8,18]. The computational domain is $0<x<1 / \cos (\alpha), 0<y<1 / \sin (\alpha)$, where $\alpha=30^{\circ}$ is the angle between $\mathrm{x}$-axis and the propagation direction of Alfven wave. Initial conditions are given as,

$$
\begin{gathered}
\rho=1, p=0.1, v_{\|}=0, B_{\|}=1, v_{\perp}=B_{\perp}=0.1 \sin [2 \pi(x \cos \alpha+y \sin \alpha)], \\
v_{\perp}=B_{\perp}=0.1 \sin [2 \pi(x \cos \alpha+y \sin \alpha)], w=B_{z}=0.1 \cos [2 \pi(c \cos \alpha+y \sin \alpha)] .
\end{gathered}
$$

Where,

$$
B_{\perp}=B_{y} \cos \alpha-B_{x} \sin \alpha, B_{\|}=B_{x} \cos \alpha+B_{y} \sin \alpha, \gamma=5 / 3 .
$$

Tablel shows the accuracy comparison of FC scheme and WENO-Z scheme. The errors of FC are much smaller than those of WENO-Z no matter in $L_{\infty}$ or $L_{1}$. The $L_{1}$ order of FC scheme approaches to sixth order.

Table1. Accuracy of Alfven wave

\begin{tabular}{llllll}
\hline Scheme & $N_{x} \times N_{y}$ & $L_{\infty}$ error & $L_{\infty}$ order & $L_{1}$ error & $L_{1}$ order \\
\hline WENO-Z & $8 \times 8$ & $3.01 \mathrm{E}-2$ & - & $1.99 \mathrm{E}-2$ & - \\
& $16 \times 16$ & $8.93 \mathrm{E}-4$ & 5.07 & $6.37 \mathrm{E}-4$ & 4.97 \\
& $32 \times 32$ & $2.38 \mathrm{E}-5$ & 5.23 & $1.68 \mathrm{E}-5$ & 5.25 \\
\hline
\end{tabular}




\begin{tabular}{llllll}
\hline & $64 \times 64$ & $7.62 \mathrm{E}-7$ & 4.96 & $4.99 \mathrm{E}-7$ & 5.07 \\
\hline FC & $8 \times 8$ & $7.27 \mathrm{E}-3$ & - & $3.26 \mathrm{E}-3$ & - \\
& $16 \times 16$ & $2.40 \mathrm{E}-4$ & 4.92 & $6.76 \mathrm{E}-5$ & 5.59 \\
& $32 \times 32$ & $2.39 \mathrm{E}-6$ & 6.65 & $7.86 \mathrm{E}-7$ & 6.42 \\
& $64 \times 64$ & $7.54 \mathrm{E}-8$ & 4.98 & $1.64 \mathrm{E}-8$ & 5.58 \\
\hline
\end{tabular}

Orszag - Tang turbulence problem. The Orszag-Tang MHD turbulence problem[19] has many significant characteristics of MHD turbulence, such as interactions of multiple shock waves generated as the vortex evolves, so it is considered as one of the standard models to validate a MHD numerical method. The computational domain is $0<x<2 \pi, 0<y<2 \pi$. Initial conditions of this problem are given as,

$$
\begin{gathered}
\rho(x, y, 0)=\gamma^{2}, \quad u(x, y, 0)=-\sin (y), v(x, y, 0)=\sin (x), \\
p(x, y, 0)=\gamma, B_{x}(x, y, 0)=-\sin (y), B_{y}(x, y, 0)=\sin (2 x) .
\end{gathered}
$$
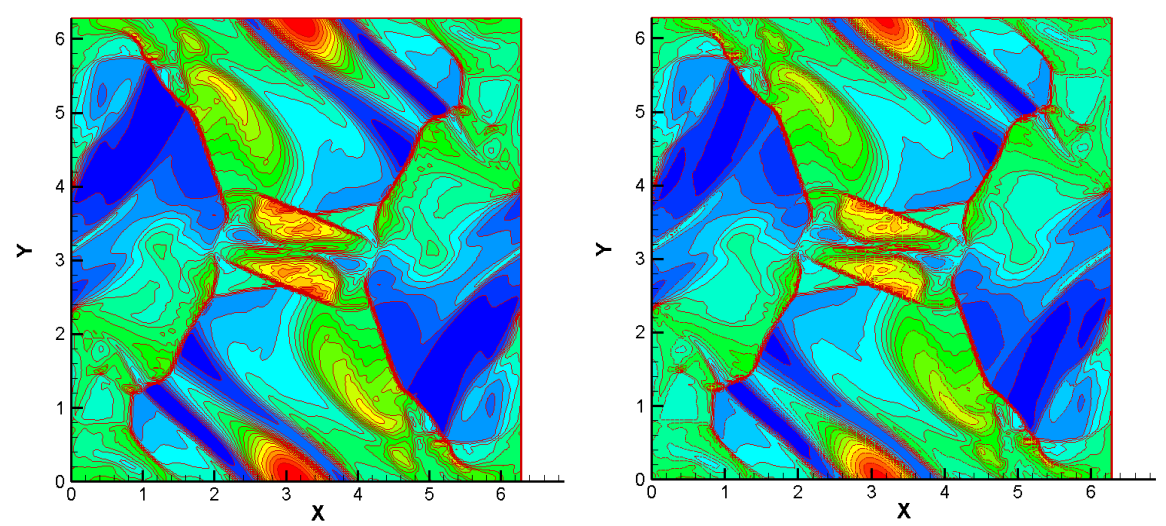

Fig.1. Density contour of Orszag-Tang MHD turbulence problem, WENO-Z and FC

For this case, how to preserve the divergence-free condition $\nabla \cdot \mathrm{B}=0$ for the magnetic field $\mathrm{B}$ is a crucial issue, same as in[8], the constrained transport(CT) method proposed by Balsara and Spicer in paper[6] is used. The mesh of $192 \times 192$ is used in this paper. Fig.1 shows the density contour of WENO-Z scheme and FC scheme at $t=3.0$. FC scheme can capture more detailed small scale structures than WENO-Z scheme. Fig. 2 is density curves comparison along $y=\pi$. It can be seen that both two schemes obtain similar distributions. The higher values at peaks and lower values at valleys obtained by FC scheme show that FC scheme has lower diffusion than WENO-Z scheme.

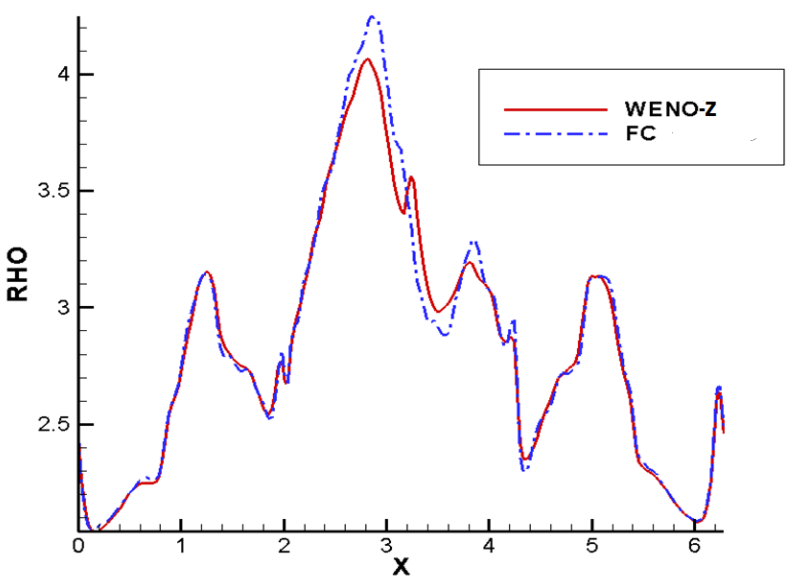

Fig.2. Density distribution along $y=\pi$ 


\section{Summary}

In this paper, a high order low diffusion shock capturing method for computing MHD flows is developed. The new method combines the E-CUSP scheme used as Riemann solver and the finite compact scheme used as reconstruction scheme. Hence, the new method avoids the complex eigenstructure of the MHD system and has the properties of the finite compact scheme. Numerical results show that the present method has the high order low diffusion in smooth regions and essentially non-oscillation near shocks.

This work is supported by the National Natural Science Foundation of China(11272325).

\section{References}

[1] M. Brio, C.C. Wu, An upwind differencing scheme for the equations of ideal magnetohydrodynamics, Journal of Computational Physics. 75 (1988) 400-422.

[2] P.L. Roe, D.S. Balsara, Notes on the eigensystem of magnetohydrodynamics, SIAM J. Appl. Math. 56 (1996)57-67.

[3] K.G. Powell, P.L. Roe, T.J. Linde, T.I. Gombosi, D.L. De Zeeuw, A Solution-Adaptive Upwind Scheme for Ideal Magnetohydrodynamics, Journal of Computational Physics. 154 (1999) 284-309.

[4] G.S. Jiang, C.C. Wu, A High-Order WENO Finite Difference Scheme for the Equations of Ideal Magnetohydrodynamics, Journal of Computational Physics. 150 (1999) 561-594.

[5] H.M. Damevin, K.A. Hoffmann, Development of a modified Runge-Kutta scheme with TVD limiters for ideal three-dimensional magnetogasdynamics, AIAA . 2001 (2001) 27-39.

[6] D.S. Balsara, D.S. Spicer, A staggered mesh algorithm using high order Godunov fluxes to ensure solenoidal magnetic fields in magnetohydrodynamics simulation, Journal of Computational Physics. 149 (1999) 270-292.

[7] S.H. Han, J. Lee, K.M. Kim, Accurate and Robust Pressure Weight Advection Upstream Splitting Method for Magnetohydrodynamics Equations, AIAA Journal. 47 (2009) 970-981.

[8] Y.Q. Shen, G.C. Zha, M.A.Huerta, E-CUSP scheme for the equations of ideal magnetohydrodynamics with high order WENO Scheme, Journal of Computational Physics. 231 (2012) 6233-6247.

[9] G.S. Jiang, C.W. Shu, Efficient implementation of weighted ENO schemes, J. Comput. Phys. 126(1996) 202-228.

[10]R. Borges, M. Carmona, B. Costa, W.S. Don, An improved weighted essentially non-oscillatory scheme for hyperbolic conservation laws, Journal of Computational Physics. 227 (2008) 3191-3211.

[11]S.H. Zhang, S.F. Jiang, C.W. Shu, Development of nonlinear weighted compact schemes with increasingly higher order accuracy, Journal of Computational Physics. 227(2008) 7294-7321

[12]E. Johnsen, J. Larsson, A.V. Bhagatwala, W. H. Cabot, P. Moin, B. J. Olson, P. S. Rawat, S. K. Shankar, B. Sjogreen, H. C. Yee, X. Zhong, and S. K. Lele, Assessment of high-resolution methods for numerical simulations of compressible turbulence with shock waves, Journal of Computational Physics. 229(2010)1213-1237

[13]Y.Q. Shen, G.W. Yang, Z. Gao, High-resolution finite compact difference schemes for hyperbolic conservation laws, J. Comput. Phys. 216(2006) 114-137. 
[14]Y.Q. Shen, G.W. Yang, Hybrid finite compact-WENO schemes for shock calculation, International Journal for Numerical Methods in Fluids. 53 (2007) 531-560.

[15]Y.Q. Shen, G.C. Zha, Generalized finite compact difference scheme for shock/complex flowfield interaction, Journal of Computational Physics. 230 (2011) 4419-4436.

[16]S.K. Lele, Compact finite difference schemes with spectral-like resolution, Journal of Computational Physics. 103(1992) 16-42

[17]C.W. Shu, O. Osher, Efficient implementation of essentially non-oscillatory shock capturing schemes, Journal of Computational Physics. 77 (1988) 439-471.

[18]G. Toth, The $\nabla \cdot \mathrm{B}=0$ constraint in shock-capturing magnetohydrodynamics codes, Journal of Computational Physics. 161 (2000) 605-652.

[19]S.A. Orszag, C.M. Tang, Small-scale structure of two-dimensional magnetohydrodynamic turbulence, J. Fluid. Mech. 90 (1979) 129-143. 


\section{Design and Manufacture in Mechanical Engineering}

10.4028/www.scientific.net/AMM.432

\section{Non-Oscillatory Finite Compact Scheme for the Equations of Ideal Magnetohydrodynamics} 10.4028/www.scientific.net/AMM.432.157

\section{DOI References}

[1] M. Brio, C.C. Wu, An upwind differencing scheme for the equations of ideal magnetohydrodynamics, Journal of Computational Physics. 75 (1988) 400-422.

http://dx.doi.org/10.1016/0021-9991(88)90120-9

[2] P.L. Roe, D.S. Balsara, Notes on the eigensystem of magnetohydrodynamics, SIAM J. Appl. Math. 56 (1996)57-67.

http://dx.doi.org/10.1137/S003613999427084X

[3] K.G. Powell, P.L. Roe, T.J. Linde, T.I. Gombosi, D.L. De Zeeuw, A Solution-Adaptive Upwind Scheme for Ideal Magnetohydrodynamics, Journal of Computational Physics. 154 (1999) 284-309.

http://dx.doi.org/10.1006/jcph.1999.6299

[4] G.S. Jiang, C.C. Wu, A High-Order WENO Finite Difference Scheme for the Equations of Ideal Magnetohydrodynamics, Journal of Computational Physics. 150 (1999) 561-594.

http://dx.doi.org/10.1006/jcph.1999.6207

[6] D.S. Balsara, D.S. Spicer, A staggered mesh algorithm using high order Godunov fluxes to ensure solenoidal magnetic fields in magnetohydrodynamics simulation, Journal of Computational Physics. 149 (1999) 270-292. http://dx.doi.org/10.1006/jcph.1998.6153

[7] S.H. Han, J. Lee, K.M. Kim, Accurate and Robust Pressure Weight Advection Upstream Splitting Method for Magnetohydrodynamics Equations, AIAA Journal. 47 (2009) 970-981.

http://dx.doi.org/10.2514/1.39375

[8] Y.Q. Shen, G.C. Zha, M.A. Huerta, E-CUSP scheme for the equations of ideal magnetohydrodynamics with high order WENO Scheme, Journal of Computational Physics. 231 (2012) 6233-6247. http://dx.doi.org/10.1016/j.jcp.2012.04.015

[10] R. Borges, M. Carmona, B. Costa, W.S. Don, An improved weighted essentially non-oscillatory scheme for hyperbolic conservation laws, Journal of Computational Physics. 227 (2008) 3191-3211. http://dx.doi.org/10.1016/j.jcp.2007.11.038

[14] Y.Q. Shen, G.W. Yang, Hybrid finite compact-WENO schemes for shock calculation, International Journal for Numerical Methods in Fluids. 53 (2007) 531-560.

http://dx.doi.org/10.1002/fld.1286

[15] Y.Q. Shen, G.C. Zha, Generalized finite compact difference scheme for shock/complex flowfield interaction, Journal of Computational Physics. 230 (2011) 4419-4436.

http://dx.doi.org/10.1016/j.jcp.2011.01.039

[17] C.W. Shu, O. Osher, Efficient implementation of essentially non-oscillatory shock capturing schemes, Journal of Computational Physics. 77 (1988) 439-471.

http://dx.doi.org/10.1016/0021-9991(88)90177-5

[18] G. Toth, TheB $=0$ constraint in shock-capturing magnetohydrodynamics codes, Journal of Computational Physics. 161 (2000) 605-652. 
http://dx.doi.org/10.1006/jcph.2000.6519

[19] S.A. Orszag, C.M. Tang, Small-scale structure of two-dimensional magnetohydrodynamic turbulence, J. Fluid. Mech. 90 (1979) 129-143.

http://dx.doi.org/10.1017/S002211207900210X 